\title{
The Reactor Antineutrino Anomaly
}

\section{Th. Lasserre*}

CEA, Irfu, SPP, Centre de Saclay, F-91191 Gif-sur-Yvette, France

E-mail: thierry.lasserre@cea.fr

Early 2011 new reactor antineutrino spectra have been provided for ${ }^{235} \mathrm{U},{ }^{239} \mathrm{Pu},{ }^{241} \mathrm{Pu}$, and ${ }^{238} \mathrm{U}$, increasing the mean flux by about 3 percent. The authors reevaluated the ratio of observed event rate to predicted rate of 19 published experiments at reactor-detector distances below $100 \mathrm{~m}$, found to be $0.943 \pm 0.023$. The deviation from unity, significant at $98.6 \%$ C.L., is now being refered as 'the reactor antineutrino anomaly'. The compatibility of our results with the existence of a fourth non-standard neutrino state driving neutrino oscillations at short distances is discussed. The combined analysis of reactor data, gallium solar neutrino calibration experiments data disfavors the no-oscillation hypothesis at $99.8 \%$ C.L. The oscillation parameters are such that $\left|\Delta m_{\text {new }}^{2}\right|>1.5 \mathrm{eV}^{2}(95 \%)$ and $\sin ^{2}\left(2 \theta_{\text {new }}\right)=0.14 \pm 0.08(95 \%)$.

The 2011 Europhysics Conference on High Energy Physics-HEP 2011,

July 21-27, 2011

Grenoble, Rhône-Alpes France

${ }^{*}$ Speaker. 


\section{Introduction}

Neutrino oscillation experiments over the last twenty years have established a picture of neutrino mixing and masses that explains the results of solar, atmospheric and reactor neutrino experiments [3]. Reactor experiments at distances below $100 \mathrm{~m}$ from the reactor core (ILL-Grenoble, Goesgen, Rovno, Krasnoyarsk, Savannah River and Bugey [4, 5, 6, 7]) have played an important role in the establishment of the current status of neutrino oscillation. The measured rate of $\bar{v}_{e}$ was found to be in reasonable agreement with that predicted from the reactor antineutrino spectra, though slightly lower than expected, with the measured/expected ratio at $0.976 \pm 0.024$, including recent revisions of the neutron mean lifetime[3] $\left(\tau_{n}=885.7 \mathrm{~s}\right)$. In preparation for the Double Chooz reactor experiment, we have re-evaluated the specific reactor antineutrino flux ( $v /$ fission), improving the electron to antineutrino data conversion [1].

\section{New Predicted Cross Section per Fission}

Fission reactors release about $10^{20} \bar{v}_{e} \mathrm{GW}^{-1} \mathrm{~s}^{-1}$, which mainly come from the beta decays of the fission products of ${ }^{235} \mathrm{U},{ }^{238} \mathrm{U},{ }^{239} \mathrm{Pu}$, and ${ }^{241} \mathrm{Pu}$. The emitted antineutrino spectrum is then given by: $S_{\text {tot }}\left(E_{v}\right)=\sum_{k} f_{k} S_{k}\left(E_{v}\right)$ where $f_{k}$ refers to the contribution of the main fissile nuclei to the total number of fissions of the $\mathrm{k}^{\text {th }}$ branch, and $S_{k}$ to their corresponding neutrino spectrum per fission. For the last 25 years the $\bar{v}_{e}$ spectra have been estimated from measurements of the total electron spectra associated with the beta decays of all fission products of ${ }^{235} \mathrm{U},{ }^{239} \mathrm{Pu}$, and ${ }^{241} \mathrm{Pu}$. Thin target foils of these isotopes were irradiated with thermal neutrons at the ILL reactor [8]. The measured spectra then had to be converted from electron to antineutrino spectra invoking a set of 30 effective beta-branches, adjusted to reproduce the total electron spectrum [10].

Recently we revisited the conversion procedure with a novel mixed-approach combining the accurate reference of the ILL electron spectra with the physical distribution of beta branches of all fission products provided by the nuclear databases [1]. This new approach provided a better handle on the systematic errors of the conversion and led to a systematic shift of about $3 \%$ in the normalization of ${ }^{235} \mathrm{U},{ }^{239} \mathrm{Pu}$, and ${ }^{241} \mathrm{Pu}$ antineutrino fluxes, respectively. This normalization shift has been attributed to the treatment of systematic effects in the original conversion of the ILL electron data. Because ${ }^{238} \mathrm{U}$ nuclei undergo fission with fast neutrons, the associated electron spectrum could not be measured in the thermal neutron flux of the ILL reactor. Therefore the ab initio summation of the $\bar{v}_{e}$ from all possible beta decays of fission products was performed to predict the neutrino spectrum [9]. In Ref. [1] we provided a new prediction with an estimated relative uncertainty of the order of $15 \%$ in the $2-8 \mathrm{MeV}$ range.

Experiments at baselines below $100 \mathrm{~m}$ reported either the ratios $(\mathrm{R})$ of the measured to predicted cross section per fission, or the observed event rate to the predicted rate. The prediction of the cross section per fission is defined as:

$$
\sigma_{f}^{\mathrm{pred}}=\int_{0}^{\infty} S_{\mathrm{tot}}\left(E_{v}\right) \sigma_{\mathrm{V}-\mathrm{A}}\left(E_{v}\right) d E_{v}=\sum_{k} f_{k} \sigma_{f, k}^{\mathrm{pred}}
$$

where the $\sigma_{f, k}^{\mathrm{pred}}$ are the predicted cross sections for each fissile isotope, $S_{\mathrm{tot}}$ is the model dependent reactor neutrino spectrum for a given average fuel composition $\left(f_{k}\right)$ and $\sigma_{\mathrm{V}-\mathrm{A}}$ is the theoretical 
cross section of reaction $\bar{v}_{e}+p \rightarrow e^{+}+n$ (see[1,2] for details). Accounting for new reactor antineutrino spectra [1] the normalization of predicted antineutrino rates, $\sigma_{f, k}^{\text {pred }}$, is shifted by $+2.5 \%$, $+3.1 \%,+3.7 \%,+9.8 \%$ for $\mathrm{k}={ }^{235} \mathrm{U},{ }^{239} \mathrm{Pu},{ }^{241} \mathrm{Pu}$, and ${ }^{238} \mathrm{U}$ respectively. In the case of ${ }^{238} \mathrm{U}$ the completeness of nuclear databases over the years largely explains the $+9.8 \%$ shift from the reference computations [9]. The new predicted cross section for any fuel composition can be computed from Eq. (2.1). By default our new computation takes into account the so-called off-equilibrium correction[1] of the antineutrino fluxes (increase in fluxes caused by the decay of long-lived fission products).

\section{Impact on past reactor neutrino experimental results}

In the eighties and nineties, experiments were performed at a few tens of meters from nuclear reactor cores at ILL, Goesgen, Rovno, Krasnoyarsk, Bugey (so called 3 and 4) and Savannah River $[4,5,6,7]$. We only consider here experiments with baselines below $100 \mathrm{~m}$ to get rid of a possible $\left(\theta_{13}, \Delta m_{31}^{2}\right)$ driven oscillation effect at Palo Verde or CHOOZ. The ratios of observed event rates to predicted event rates (or cross section per fission), $R=N_{\mathrm{obs}} / N_{\text {pred }}$, are summarized in Table 1. The observed event rates and their associated errors are unchanged with respect to the publications, the predicted rates are reevaluated separately in each experimental case. We observe a general systematic shift more or less significantly below unity. These reevaluations unveil a new reactor antineutrino anomaly [2]. In order to quantify the statistical significance of the anomaly we can compute the weighted average of the ratios of expected over predicted rates, for all short baseline reactor neutrino experiments (including their possible correlations). We consider

Table 1: $\quad N_{\text {obs }} / N_{\text {pred }}$ ratios based on old and new spectra. The err column is the total error published by the collaborations including the error on $S_{\text {tot }}$, the corr column is the part of the error correlated among experiments.

\begin{tabular}{|c|c|c|c|c|c|c|c|c|c|c|c|c|}
\hline$\#$ & result & Det. type & $\tau_{n}(\mathrm{~s})$ & ${ }^{235} \mathrm{U}$ & ${ }^{239} \mathrm{Pu}$ & ${ }^{238} \mathrm{U}$ & ${ }^{241} \mathrm{Pu}$ & old & new & $\operatorname{err}(\%)$ & $\operatorname{corr}(\%)$ & $\mathrm{L}(\mathrm{m})$ \\
\hline 1 & Bugey-4 & ${ }^{3} \mathrm{He}+\mathrm{H}_{2} \mathrm{O}$ & 888.7 & 0.538 & 0.328 & 0.078 & 0.056 & 0.987 & 0.942 & 3.0 & 3.0 & 15 \\
2 & ROVNO91 & ${ }^{3} \mathrm{He}+\mathrm{H}_{2} \mathrm{O}$ & 888.6 & 0.614 & 0.274 & 0.074 & 0.038 & 0.985 & 0.940 & 3.9 & 3.0 & 18 \\
\hline 3 & Bugey-3-I & ${ }^{6} \mathrm{Li}-\mathrm{LS}$ & 889 & 0.538 & 0.328 & 0.078 & 0.056 & 0.988 & 0.946 & 4.8 & 4.8 & 15 \\
4 & Bugey-3-II & ${ }^{6} \mathrm{Li}-\mathrm{LS}$ & 889 & 0.538 & 0.328 & 0.078 & 0.056 & 0.994 & 0.952 & 4.9 & 4.8 & 40 \\
5 & Bugey-3-III & ${ }^{6} \mathrm{Li}-\mathrm{LS}$ & 889 & 0.538 & 0.328 & 0.078 & 0.056 & 0.915 & 0.876 & 14.1 & 4.8 & 95 \\
\hline 6 & Goesgen-I & ${ }^{3} \mathrm{He}+\mathrm{LS}$ & 897 & 0.620 & 0.274 & 0.074 & 0.042 & 1.018 & 0.966 & 6.5 & 6.0 & 38 \\
7 & Goesgen-II & ${ }^{3} \mathrm{He}+\mathrm{LS}$ & 897 & 0.584 & 0.298 & 0.068 & 0.050 & 1.045 & 0.992 & 6.5 & 6.0 & 45 \\
8 & Goesgen-II & ${ }^{3} \mathrm{He}+\mathrm{LS}$ & 897 & 0.543 & 0.329 & 0.070 & 0.058 & 0.975 & 0.925 & 7.6 & 6.0 & 65 \\
9 & ILL & ${ }^{3} \mathrm{He}+\mathrm{LS}$ & 889 & $\simeq 1$ & - & - & - & 0.832 & 0.802 & 9.5 & 6.0 & 9 \\
\hline 10 & Krasn. I & ${ }^{3} \mathrm{He}+\mathrm{PE}$ & 899 & $\simeq 1$ & - & - & - & 1.013 & 0.936 & 5.8 & 4.9 & 33 \\
11 & Krasn. II & ${ }^{3} \mathrm{He}+\mathrm{PE}$ & 899 & $\simeq 1$ & - & - & - & 1.031 & 0.953 & 20.3 & 4.9 & 92 \\
12 & Krasn. III & ${ }^{3} \mathrm{He}+\mathrm{PE}$ & 899 & $\simeq 1$ & - & - & - & 0.989 & 0.947 & 4.9 & 4.9 & 57 \\
\hline 13 & SRP I & Gd-LS & 887 & $\simeq 1$ & - & - & - & 0.987 & 0.952 & 3.7 & 3.7 & 18 \\
14 & SRP II & Gd-LS & 887 & $\simeq 1$ & - & - & - & 1.055 & 1.018 & 3.8 & 3.7 & 24 \\
\hline 15 & ROVNO88-1I & ${ }^{3} \mathrm{He}+\mathrm{PE}$ & 898.8 & 0.607 & 0.277 & 0.074 & 0.042 & 0.969 & 0.917 & 6.9 & 6.9 & 18 \\
16 & ROVNO88-2I & ${ }^{3} \mathrm{He}+\mathrm{PE}$ & 898.8 & 0.603 & 0.276 & 0.076 & 0.045 & 1.001 & 0.948 & 6.9 & 6.9 & 18 \\
17 & ROVNO88-1S & Gd-LS & 898.8 & 0.606 & 0.277 & 0.074 & 0.043 & 1.026 & 0.972 & 7.8 & 7.2 & 18 \\
18 & ROVNO88-2S & Gd-LS & 898.8 & 0.557 & 0.313 & 0.076 & 0.054 & 1.013 & 0.959 & 7.8 & 7.2 & 25 \\
19 & ROVNO88-3S & Gd-LS & 898.8 & 0.606 & 0.274 & 0.074 & 0.046 & 0.990 & 0.938 & 7.2 & 7.2 & 18 \\
\hline
\end{tabular}


the following experimental rate information: Bugey-4 and Rovno91, the three Bugey-3 experiments, the three Goesgen experiments and the ILL experiment, the three Krasnoyarsk experiments, the two Savannah River results (SRP), and the five Rovno88 experiments. $\vec{R}$ is the corresponding vector of 19 ratios of observed to predicted event rates. We assume a $2.0 \%$ systematic uncertainty fully correlated among all 19 ratios in result of the common normalization uncertainty of the beta-spectra measured in [8]. In order to account for the potential experimental correlations, we fully correlated some of the experimental errors [2]. With our new reevaluation we obtain the mean ratio is $\mu=0.943 \pm 0.023$, corresponding to a $-2.2 \sigma$ effect (while a simple calculation assuming normality would lead to $-2.4 \sigma$ ). Clearly the new spectra induce a statistically significant deviation from the expectation. In the following we define an experimental cross section $\sigma_{f}^{\text {ano }}=0.943 \times \sigma_{f}^{\text {pred,new }} 10^{-43} \mathrm{~cm}^{2} /$ fission. Assuming the correctness of $\sigma_{f}^{\text {pred,new }}$ the anomaly could still be explained by a common bias in all reactor neutrino experiments, but this is unlikely since the measurements used different detection techniques. The other possible explanation of the anomaly is based on a real physical effect.

We used shape information from the Bugey-3 and ILL published data [5, 4] for our combined analysis. From the analysis of the shape of their energy spectra at different source-detector distances [5, 6], the Goesgen and Bugey-3 measurements exclude oscillations such that $0.06<\Delta m^{2}<$ $1 \mathrm{eV}^{2}$ for $\sin ^{2}(2 \theta)>0.05$. We used Bugey-3's $40 \mathrm{~m} / 15 \mathrm{~m}$ ratio data from [5] as it provides the best limit.

\section{The fourth neutrino hypothesis}

The reactor antineutrino anomaly could be explained through the existence of a fourth nonstandard neutrino, corresponding in the flavor basis to a sterile neutrino $v_{s}$ (see [3] and references therein) with a large $\Delta m_{\text {new }}^{2}$ value. For simplicity we restrict our analysis to the $3+1$ four-neutrino scheme in which there is a group of three active neutrino masses separated from an isolated neutrino mass, such that $\left|\Delta m_{\text {new }}^{2}\right| \gg 10^{-2} \mathrm{eV}^{2}$. The latter would be responsible for very short baseline reactor neutrino oscillations. For energies above the inverse beta decay threshold and baselines below $100 \mathrm{~m}$, we adopt the approximated oscillation formula:

$$
P_{e e}=1-\sin ^{2}\left(2 \theta_{\text {new }}\right) \sin ^{2}\left(\frac{\Delta m_{\text {new }}^{2} L}{4 E_{\bar{v}_{e}}}\right)
$$

where active neutrino oscillation effects are negligible at these short baselines.

We combined our results with previously quoted anomalies affecting other short baseline electron neutrino experiments Gallex, Sage and MiniBooNE, reviewed in Ref. [11]. We reanalyzed the Gallex and Sage calibration runs with ${ }^{51} \mathrm{Cr}$ and ${ }^{37} \mathrm{Ar}$ radioactive sources emitting $\sim 1 \mathrm{MeV}$ electron neutrinos. [12], following the methodology developed in Ref. [13, 11]. We also reanalyzed the MiniBooNE electron neutrino excess assuming the very short baseline neutrino oscillation explanation of Ref. [11]. The no-oscillation hypothesis is disfavored at 99.8\% C.L. The significance is dominated by the gallium and reactor data. Allowed regions in the $\sin ^{2}\left(2 \theta_{\text {new }}\right)-\Delta m_{\text {new }}^{2}$ plane are displayed in Fig. 1, together with the marginal $\Delta \chi^{2}$ profiles for $\left|\Delta m_{\text {new }}^{2}\right|$ and $\sin ^{2}\left(2 \theta_{\text {new }}\right)$. The combined fit leads to the following constraints on oscillation parameters: $\left|\Delta m_{\text {new }}^{2}\right|>1.5 \mathrm{eV}^{2}$ (95\% C.L.) and $\sin ^{2}\left(2 \theta_{\text {new }}\right)=0.14 \pm 0.08$ (95\% C.L.). 


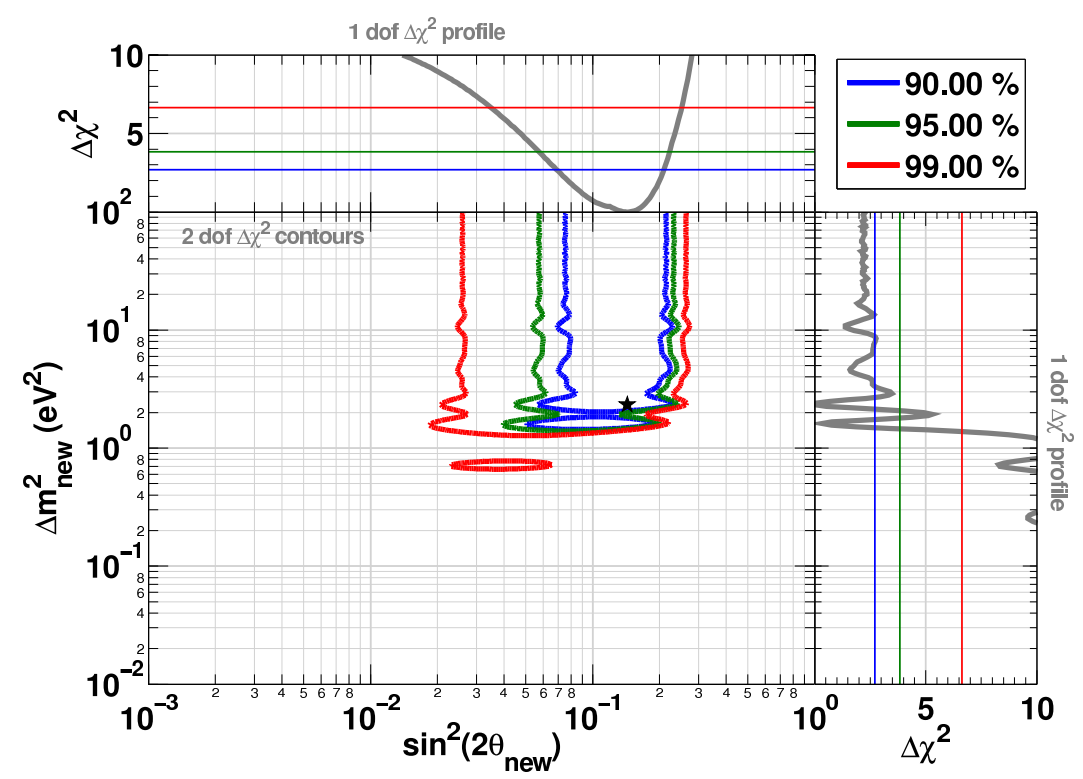

Figure 1: Allowed regions in the $\sin ^{2}\left(2 \theta_{\text {new }}\right)-\Delta m_{\text {new }}^{2}$ plane from the combination of reactor neutrino experiments, Gallex and Sage, MiniBooNE. The data are well fitted by the $3+1$ neutrino hypothesis.

The reactor antineutrino anomaly could be explained through the existence of a fourth non-standard neutrino, corresponding in the flavor basis to a sterile neutrino $v_{s}$ with a large $\Delta m_{\text {new }}^{2}$ value. This hypothesis must be tested experimentaly.

\section{References}

[1] Th. A. Mueller, et al., arXiv:1101.2663; Phys. Rev. C83, 054615, (2011).

[2] G. Mention, et al., arXiv:1101.2755; Phys. Rev. D83, 073006, (2011).

[3] K. Nakamura et al., (Particle Data Group), J. Phys. G37, 075021 (2010).

[4] H. Kwon et al., Phys. Rev. D24, 1097, (1981).

[5] B. Achkar et al., Nucl. Phys. B434, 503, (1995).

[6] G. Zacek et al., Phys. Rev. D34, 2621, (1986).

[7] Y. Déclais et al., Phys. Lett. B 338, 383, (1994); A.I. Afonin et al., JETP94, 1-17, (1988); V. Kuvshinnikov et al., JETP54 N5, 259, (1991); G.S. Vidyakin et al., JETP93, 424-431, (1987); G.S. Vidyakin et al., JETP59, 390, (1994); Z.D. Greenwood et al., Phys. Rev. D53, 11, (1996).

[8] K. Schreckenbach et al., Phys. Lett. B 99, 251, (1981); K. Schreckenbach et al., Phys. Lett. B 160, 325, (1985); F. von Feilitzsch, A. A. Hahn and K. Schreckenbach, Phys. Lett. B 118, 162, (1982);

A. A. Hahn et al., Phys. Lett. B 218, 365, (1989).

[9] B. R. Davis et al., Phys. Rev. C19, 2259, (1979). P. Vogel et al., Phys. Rev. C24, 1543, (1981).

[10] P. Vogel, Phys. Rev. D29, 1918, (1984).

[11] C. Giunti, M. Laveder, Phys. Rev. D82, 053005, (2010). 
[12] W. Hampel et al. (GALLEX), Phys. Lett. B 420, 114, (1998); J. N. Abdurashitov et al. (SAGE), Phys. Rev. C80, 015807, (2009).

[13] C. Giunti, M. Laveder, arXiv:1006.3244v2.

[14] A. A. Aguilar-Arevalo et al., Phys. Rev. Lett. 102, 101802, (2009). 